Etnográfica

Revista do Centro em Rede de Investigação em

Antropologia

vol. 17 (3) | 2013

Vol. $17(3)$

\title{
En forêt, la musique : entre inquiétude et sentiment d'intimité (Goğğam, Ethiopie)
}

In the forest, music : between restlessness and intimacy feelings (Goğğam, Ethiopia)

\section{Katell Morand}

\section{(2) OpenEdition}

\section{Journals}

Édition électronique

URL : https://journals.openedition.org/etnografica/3291

DOI : 10.4000/etnografica.3291

ISSN : 2182-2891

Éditeur

Centro em Rede de Investigação em Antropologia

Édition imprimée

Date de publication : 30 octobre 2013

Pagination : 561-579

ISSN : 0873-6561

\section{Référence électronique}

Katell Morand, « En forêt, la musique : entre inquiétude et sentiment d'intimité (Goğğam, Ethiopie) », Etnográfica [En ligne], vol. 17 (3) | 2013, mis en ligne le 30 octobre 2013, consulté le 09 février 2022 URL : http://journals.openedition.org/etnografica/3291; DOI : https://doi.org/10.4000/etnografica. 3291

\section{(c) (1) (9)}

Etnográfica is licensed under a Creative Commons Attribution-NonCommercial 4.0 International License. 


\section{En forêt, la musique : entre inquiétude et sentiment d'intimité (Goğğam, Ethiopie)}

\section{Katell Morand}

Sur les plateaux éthiopiens du Goğğam, les bergers passent une grande partie de leur temps à siffler, chanter ou jouer de la flûte. Ces forêts sont des lieux à la marge de la vie sociale, peuplées de bandits. La musique peut être à la fois considérée comme un outil de contrôle de cet espace sauvage, et comme un risque de se faire entendre des rôdeurs malveillants de la forêt. La musique est donc un acte de bravoure en même temps qu'un intense moment de solitude et de vulnérabilité.

MOTS-CLÉS : Ethiopie, chant, forêt, intimité, berger.

In the forest, music: between restlessness and intimacy feelings (Groğğam, Ethiopia) - In the Ethiopian Highlands of Goğğam, the herders spend most of their time whistling, singing, and playing the flute. These forests are social marginal lands and music can be considered as a tool for controlling the "wild" but performing music appears risky, for the herder reveals his position to potential thieves. Music is therefore an act of bravery in an unfriendly environment, a moment of both solitude and vulnerability.

KEYWORDS: Ethiopia, singing, forest, intimité, herder.

MORAND, Katell (kmorand@u.washington.edu) - School of Music, University of Washington, EUA. 
Jrä t’arañ čakkaw ərä t’orañ duru

Lantäm yš̌alähal bəča kämadäru
Ah Forêt, appelle-moi, ah Nature, appelle-moi !

Pour toi aussi cela vaut mieux, que de passer seule la nuit

“C'EST BIEN LE SON DE LA FORET". GANNATU AVAIT UN TEMPS PARU songeur ; mais en reposant les écouteurs, son visage s'éclaira d'un sourire de satisfaction : le chant lui plaisait. Bien que Gännätu soit devenu trop âgé pour garder ses bêtes en forêt, l'enregistrement de la veille, effectué à l'enclos de nuit installé tout près de la lisière, avait capté les bruits du fleuve, les stridulations des insectes, et les mouvements légers du bétail derrière une voix assourdie par le pan relevé de son vêtement.

Faire de la musique en forêt est extrêmement répandu dans cette région des haut-plateaux Amhara du Goğğam, en bordure du fleuve Abbay (ou Nil Bleu). Presque tous les hommes s'y rendent à intervalles réguliers en quête de pâturages pour leur bétail. Durant leurs déambulations solitaires ou lors des veillées nocturnes, ils chantent, jouent de la flûte et sifflent un mélange hétéroclite d'airs entendus ailleurs et en d'autres temps : lors des fêtes de village, des cérémonies funéraires ou des travaux des champs. Ces airs - poèmes chantés et autres motifs mélodiques - demeurent très fortement attachés à leurs contextes initiaux; ils sont reconnaissables presque instantanément et conservent les mêmes dénominations (leur genre, par exemple). Pourquoi alors Gännätu, comme tant d'autres, s'empresse-t-il de les reconnaître, pour affirmer son appréciation, comme des "sons de la forêt"?

Ce que nous allons voir dans cet article, c'est qu'au-delà de leur certaine qualité esthétique - la voix mêlée aux sons de la nature - se joue une relation particulière entre la pratique musicale et l'espace de la forêt: dans le trouble de la distance et la séparation, entre la maîtrise et la perte de contrôle, et les interstices "sauvages" et intimes de la vie sociale.

\section{DES MARGES INQUIETANTES}

Au village, l'eau et la forêt sont d'une présence insistante. Le grondement du fleuve, toile de fond sonore de la vie quotidienne, parvient jusqu'aux flancs des collines où se perchent les habitations ; et les regards, où qu'ils portent, croisent le cours ample du fleuve, les torrents qui le rejoignent à travers la plaine, ou bien les arbres dont le vert profond tranche avec la nudité des plateaux. Rien n'est plus familier que ces dur ou dän ("nature sauvage”) qu'il faut longer mais rarement traverser - pour se rendre au marché ou visiter des parents. Rien n'est plus inquiétant non plus : car il s'agit là d'un “désert” (bäräha), un lieu 
non habité, où ne se trouvent "que les arbres, l'eau, les animaux sauvages, et les vaches", ${ }^{1}$ et que tout oppose à la société villageoise.

De fait, ces espaces "sauvages" sont à part. Bien qu'ils appartiennent administrativement au territoire de la commune, ${ }^{2}$ ils sont en marge de ce que les habitants nomment leur "pays" (agär), un terme qui a la même indétermination - village, région, ou nation - et la même connotation affective qu'en français. Le "pays", c'est d'abord la paroisse (däbər) dont l'église représente le centre à la fois géographique et symbolique. ${ }^{3}$ Située au point le plus élevé, à une heure de marche tout au plus des habitations, elle domine le plateau et les plaines alentour. Forêt et fleuve, sources de croyances plus ou moins tolérées ou intégrées par la religion chrétienne, semblent à l'écart de son influence : à l'Abbay, on présente régulièrement des offrandes ; et on ne manque pas de se prémunir des démons, saytan, qui logeraient tout au fond de l'eau ou au sommet des plus grands arbres.

Mais c'est aussi en repoussant la "nature sauvage" que la paroisse s'est constituée. Il y a moins d'un siècle, en effet, la majeure partie de cette vallée et des montagnes qui l'enserrent étaient encore recouvertes d'une végétation dense. Pour reprendre les mots de James Bruce, qui traversa la région en 1770, ce n'était là qu' "un pays couvert de bois, sauvage et inhabité". ${ }^{4}$ Et si l'on se fie aux souvenirs des villageois qui l'ont connue dans les années 1950, la forêt s'étendait encore très loin, recouvrant presque toute la plaine. Il ne fallut que quelques décennies de pression démographique, de défrichage, et de ramassage du bois pour qu'elle diminue considérablement, leurs pères et grands-pères la repoussant ainsi dans le fond le plus inaccessible de la vallée. En cet endroit, en effet, le cours du fleuve se divise en une multitude de bras étroits, parcourus de cascades et de courants violents qu'on ne peut traverser, à la nage, qu'en des points bien précis. Cette division du fleuve a créé d'innombrables îles, certaines minuscules, les plus grandes de l'ordre d'une dizaine d'hectares, et toutes d'accès difficile, y compris en saison sèche. Et chaque année, à la saison des pluies, les chemins deviennent impraticables : le fleuve sort de son lit, et les flots rapides rendent les traversées dangereuses; des torrents dévalent les pentes des collines et l'eau envahit les berges, la forêt, et une grande partie de vallée, transformée en vaste marécage, isolant les hameaux durant deux à trois mois.

Dans ces conditions, il n'est pas étonnant que ces espaces ne soient pas vraiment intégrés au territoire administratif, bien que celui-ci soit par ailleurs extrêmement quadrillé et contrôlé. Les moyens sont d'ailleurs extrêmement limités. Dans la plupart des villages il n'existe aucune police à proprement

1 Commentaire d'Abäba T'asaw.

2 Le qäbäle, la plus petite unité administrative de l'Etat éthiopien.

3 La quasi-totalité de la population de cette région appartient à l'Eglise orthodoxe éthiopienne.

4 Ce sont, plus précisément, les mots d'un ami de l'explorateur (Bruce 2004 [1790-92]: 93). 
parler. Le respect des lois est assuré par des gardes ou “juges”, désignés par le comité exécutif de la commune et qui, armés en permanence, effectuent des rondes régulières, délivrent des rapports oraux, et rendent occasionnellement des jugements en cas de délit. Mais aucun garde ne surveille la forêt ; ils sont impuissants et surtout, comme les habitants ne manquent de répéter avec une certaine emphase, "ils ont peur" - une peur que l'on retrouve à tous les niveaux de discours, qu'il s'agisse des conversations les plus ordinaires comme des poèmes chantés :

$\begin{aligned} \begin{array}{r}\text { Asar näw mäkära näw abbay } \\ \text { bälelit hedän bäčälläma }\end{array} & \begin{array}{l}\text { Quelle souffrance, quel malheur } \\ \text { quand nous sommes allés de nu } \\ \text { l'Abbay, dans l'obscurité }\end{array} \\ \text { Wallab wallab alä yäsänel t’ala } & \begin{array}{l}\text { Elles se balançaient de ci de là, } \\ \text { les ombres des palmiers }\end{array}\end{aligned}$

Que craignent-ils donc? Probablement moins les animaux (hyènes ou léopards) ou des êtres surnaturels que des dangers très humains. Car la violence semble indissociable des lieux. Là-bas, me répétait-on, se réfugient les "voleurs" (leba) ou "bandits" (šofta), ces gens qui "mangent souvent de la viande" - celle du bétail qu'ils enlèvent de nuit ou parfois en plein jour, lorsqu'il échappe à la surveillance de ses gardiens - sur lesquels j'entendais des histoires plus invraisemblables les unes que les autres : comment auraient pu vivre en cet endroit les cinq hommes armés, de petite taille et au regard terrifiant, que l'un jurait avoir aperçus en se penchant pour boire au fleuve ? D'où venaient les hommes inconnus qui, comme on me le racontait avec force détails, avaient menacé telle jeune femme alors qu'elle ramassait du bois en compagnie d'une de ses amies? Dans tous les cas, les rencontrer exposerait à une confrontation directe et violente : "ils frappent, ils tuent", m'affirmait-on, en soulignant la parole d'un geste d'égorgement particulièrement expressif.

Cette violence est aussi celle des ennemis personnels. Car ce sont "aux vaches", dans ces lieux non contrôlés, que se jouent les conflits personnels entre individus, les rancunes parfois longtemps réprimées et les vengeances à accomplir, lorsqu'un sang doit être "rendu". Les histoires ne manquent pas. Ainsi, on me raconta qu'en 2004, le premier mari d'une de nos voisines avait été tué alors qu'il gardait ses vaches ; et à peu près à la même époque, un autre jeune homme fut abattu de nuit au bärät, l'enclos provisoire établi en forêt ou en plein champ. Son cousin, alors un enfant, dormait avec lui sous la même couverture ; un homme armé surgit et écarta l'enfant, qui cria. Puis il tira à 
bout portant sur le jeune homme, que l'enfant vit lentement mourir. Devenu adolescent, il continuait à revivre régulièrement la scène dans ses rêves et était extrêmement angoissé à l'idée de passer la nuit à l'enclos. La nature, de façon générale, l'inquiétait : au début des années 2000, un de ses oncles avait abattu un voleur qui s'était glissé dans son enclos et si aucune vengeance n'avait encore eu lieu, tous étaient depuis des cibles potentielles et il évitait, m’expliqua-t-il, de s'aventurer le soir sur les sentiers déserts.

\section{MAITRISER L'ESPACE : LE SON COMME OUTIL}

Dans ces conditions, la musique apparaît en premier lieu comme un savoirfaire. La garde en forêt nécessite en effet tout un éventail de compétences techniques. Y emmener son troupeau nécessite de faire franchir à chaque bête, une par une, jusqu'à trois ou quatre de ces bras à la nage, d'où une dépense considérable d'énergie et le risque élevé de perdre des animaux. Une fois sur place, il faut encore s'orienter dans un labyrinthe d'espaces fragmentés où la visibilité est considérablement réduite. De nombreux toponymes (par exemple $t^{\prime}$ ’ğa mätabiya, "là où les veaux se lavent", Mälaquse Bet, "la maison du moine", ou encore Gännät qurt', "l'île du paradis") quadrillent le territoire et permettent un repérage assez précis. Le reste se fait en grande partie d'oreille. La forêt bruisse en effet d'une multitude de mouvements. Ce sont ceux de l'eau, qui court en d'innombrables torrents et cascades, parfois au ras du sol, dans les roseaux et les grandes herbes. Ce sont aussi ceux des animaux : les plus rares comme les léopards, les petites panthères noires, les antilopes, ou encore les pythons imposants ; les hippopotames et les crocodiles, aperçus depuis les berges ; les oiseaux et les singes grivets peuplant ces grands arbres qui s'élèvent jusqu'à 15 ou 20 mètres ; et enfin les hyènes, phacochères, ou babouins qui peuplent un entremêlement d'arbustes, de fougères, et de lianes.

Le troupeau doit être surveillé et protégé presque à chaque instant : car il suffit de quelques minutes d'inattention pour qu'une vache disparaisse ou se noie dans un courant violent. La tâche est d'autant plus difficile qu'elles sont la plupart du temps hors de vue, cachées par les arbres et par les épais fourrés d'épineux, et que l'absence de chemins tracés les incite à la dispersion. En effet, à l'exception de quelques clairières, vestiges de campements passés, cette végétation est d'une telle densité qu'elle impose des déplacements constants et une séparation des gardiens dès le début de la journée. Ils ne donnent l'impression ni de guider leurs vaches ni même de les suivre, mais de tenter en permanence de les rattraper. Ils louvoient dans la végétation, se penchent, s'accroupissent et courent ainsi, sans jamais abattre ni même écarter les branches qui le gênent. Et toujours ils savent, semble-t-il, où se trouvent chacune de leurs vaches.

Certaines cependant disparaissent. Il faut alors partir à leur recherche, au risque de perdre les autres de vue. Certaines de nos journées peuvent être 
entièrement consacrées à retrouver des bêtes disparues en début de la matinée car pendant les quelques dizaines de minutes nécessaires, chaque jour, au nettoyage du campement, leurs gardiens n'ont d'autre choix que de les laisser à elles-mêmes. Mais c'est surtout en fin d'après-midi que la tension se fait sentir. Dès que la luminosité baisse, les vaches doivent être ramenées au campement pour éviter les attaques des hyènes. Les gardiens convergent alors pour rassembler l'ensemble du troupeau, retrouver les retardataires, et avancer rapidement ; et certains jours, il s'en faut de peu qu'ils n'abandonnent leurs recherches.

Dans ces conditions, les sifflets - fučät, ou yälam fučät "les sifflets des vaches" - sont le meilleur outil à disposition du gardien pour surveiller et appeler son troupeau. Ils font partie de leur savoir-faire, au même titre que le maniement du bâton et la connaissance précise du terrain. Conçu, tout à fait explicitement, comme un moyen de communication avec les vaches (qui l' “écoutent"), le fuč ät leur est également réservé, à l'exclusion de tout autre usage. Ils permettent de guider : mêlés à des interjections (nah! "viens!"), ils sont utilisés pour rappeler un animal retardataire, maintenir sa direction et l'inciter à avancer. Certains gardiens m'ont déclaré que chaque motif mélodico-rythmique sifflé correspondait à un signal et à une fonction déterminée. C'est une affirmation que l'analyse musicale ne semble pas confirmer : certes on reconnaît facilement les appels sifflés - qui jouent le même rôle que des exclamations parlées - car ils ont tendance à suivre un mouvement ascendant et le plus souvent en souffle inspiré. Mais les autres motifs de fučä aucune catégorisation bien nette : ils sont très variables selon les individus, et consistent en une exploration, plutôt en mouvements conjoints, de l'échelle pentatonique (c'est-à-dire à cinq notes) répandue dans toute cette région.

De fait, l'intérêt du fuč ät semble plutôt dans la relation aux animaux et à l'espace, qu'il contribue à créer. Ces sifflements sont produits surtout pour "faire plaisir" aux vaches, dont on dit qu'elles en apprécient le son (däsilačäwal, "cela leur fait plaisir"), broutent plus et se reposent mieux. Ils sont la matérialisation sonore du souci et de l'affection de leur gardien, indépendante de la vue et du toucher, et lui permettent de maintenir le contact même lorsqu'il ne les voit plus. Dans un espace où la tension se mêle à l'ennui des jours identiques, les sifflets "pour les vaches" se superposent ainsi aux bruits de la nature pour créer le fond sonore de la solitude. Quasiment sans interruption tout au long de la journée, ils ne cessent que pour laisser la place à la flûte et au chant, que ce soit en journée lors des quelques arrêts qui rythment le parcours ou une fois la nuit tombée : après avoir rejoint l'enclos hâtivement consolidé de buissons d'épineux, et s'être réchauffés en partageant leur repas frugal.

Les sifflements marquent ainsi le déplacement de l'individu et de son troupeau. Ils signalent une présence humaine dans des lieux dont la caractéristique principale est - comme nous l'avons vu - de ne pas avoir subi cette transformation nécessaire pour être cultivés, habités et intégrés. Le fuč ’ät apparaît donc 
comme un équivalent musical de la toponymie, c'est-à-dire une appropriation de l'espace. Pour reprendre une belle expression de Michel De Lannoy (1994), dans un article à propos de chants de labours, on pourrait dire que le fučc ät est une "résonance en mouvement". Il correspondrait donc à ces caractéristiques qui sont, selon Gerd Spittler (2001: 228), celles de toutes les musiques de bergers : sinon un moyen de se maintenir dans l'humanité, ${ }^{6}$ du moins l'instrument d'une appropriation et d'un contrôle de l'espace permettant de garantir la sécurité du troupeau et la sienne propre.

Mais il faut se garder de généraliser cet aspect à l'ensemble de la pratique musicale en forêt. Il me semble que s'y mêlent en effet un certain nombre d'enjeux et de conséquences, et en tout premier lieu ceux d'une communication paradoxale et non contrôlée avec le monde sauvage.

\section{QUI CONTROLE QUOI?}

Ceci apparaît très clairement dans le jeu de la flûte dite wašsnt ou encore šämbäqo (le “roseau”). Répandu dans toute l'Éthiopie du Nord, du Sud et de l'Ouest, cet instrument, joué presque uniquement par les bergers, est étroitement associé à la forêt. De facture très simple, il est fabriqué à partir de roseaux du fleuve et se compose de quatre à cinq segments, percés de quatre trous. Il est surtout joué de nuit, dans l'enclos. En journée, il ne faut pas jouer, me répétait-on souvent, sous peine d'attirer l'un des léopards de l'île ; car "quand il entend, il vient".

Simon Messing (1957), qui a rapporté dans sa thèse de doctorat des propos similaires, voit dans cette flûte un outil de prise de pouvoir magique sur le léopard, qu'il s'agisse de se protéger (en le maintenant à distance) ou de l'attirer dans un piège - une prise de pouvoir effective par le sonore (des combinaisons spécifiques de sons) ainsi que par le contact avec l'instrument (puisqu'il doit ensuite être jeté dans la fosse). Le second à mentionner l'existence de "charme" est Ashenafi Kebede, qui retranscrit la "légende populaire" de cette manière :

"A washint performing bandit in the bushes was confronted by a fierce leopard. The animal trailed the bandit by the melody of the washint. When the bandit stopped playing, the leopard appeared restless and ferocious and nugded the bandit with its paw (so as to say 'play'). As soon as the bandit recomenced to play, the leopard dropped its fiendish stare to the ground and seemed to be absorbed.

The animal did not realise it took a lot of breath to blow on the washint. The bandit died after and exhaustive bout of playing, and the leopard, 
traditionally considered a noble animal respectful of the dead, withdrew and departed" (Ashenafi Kebede 1971: 157).

Selon Ashenafi Kebede, le léopard est attiré non par piège mais en raison de sa mélomanie, au sens fort du terme, et ce goût irrépressible pour le son de la flûte s'avère fatal non pour lui mais pour le musicien. Cette deuxième version est, au contraire de la première, extrêmement proche de ce que disent les Goğğamés.

Le meilleur exemple dont je dispose est un récit que j'ai recueilli lors de mon tout premier terrain, à l'automne 2003. A l'époque, je ne parlais que quelques mots d'amharique et j'avais demandé à Täsämma Aläm, un jeune lycéen revenu pour quelques jours chez lui, de mener l'entretien pour moi avec T'äbəqäw, un joueur de wašənt. ${ }^{7}$ C'est donc Täsämma qui mène l'entretien, à partir des questions préalables que je lui ai fournies, sans que j'intervienne d'aucune manière dans leur conversation :

Täsämma - Alors, pour commencer... quand le léopard entend le wašənt, en quoi consiste leur association?

T'äbəqäw - A chaque fois qu'il entend, il vient. Lui. Toi en revanche, tu ne le vois pas. Nous, nous ne le voyons pas et nous continuons tranquillement à jouer du wašənt. Quand nous jouons du wašənt, lui, à chaque fois qu'il entend, il faut qu'il vienne, tout simplement.

Täsämma - Oui, il vient.

T’äbəqäw - Quand il arrive là, à côté, il apparaît subitement. Il apparaît subitement et quand tu fais silence le jeu s'interrompt. Quand le jeu s'interrompt, “ joue!”...

Täsämma - Il s'excite !

T'äbəqäw - Il gratte.

Täsämma - Il gratte. Avec la patte, donc.

T'äbəqäw - Avec la patte. C'est ce qu'il te fait. Après que tu t'arrêtes.

Täsämma - Quand tu t'arrêtes.

T'äbəqäw - Quand tu t'arrêtes. Mais quand tu joues, il écoute assis en silence.

T’äbəqäw décrit ici les relations entre la flûte et le léopard sous la forme d'un énoncé de type vérité générale en trois temps :

1. “À chaque fois qu'il entend, il faut qu'il vienne" : l'action de la flûte sur l'animal est présentée comme quasiment mécanique. Une force, ou un désir - on ne sait pas trop - s'empare du léopard et l'oblige à venir, 
sans qu'aucune intentionnalité (ni celle du joueur, ni celle de l'animal, à supposer que cette dernière existe) n'entre en ligne de compte.

2. “Il apparaît subitement", et s'installe à côté du joueur.

3. De sa patte, il le force à continuer. Ce détail, impressionnant, fait toute la puissance de l'image. Comme me l'affirmait un joueur : "Il aime la flûte, il faut qu'il écoute”. D’ailleurs, “c'est pour cela que peu de gens en jouent, ils ont peur" ; du moins, “c'est ce que nous disaient nos pères" ; peutêtre la peur expliquait pourquoi les hommes de cette localité, bien qu'ils possèdent presque tous un instrument, n'en jouent qu' "un petit peu”, selon leurs propres dires, et qu'aucun d'entre eux ne soit bon joueur.

On me disait qu'il s'agissait d'un fait, d'une "vérité" et, certainement pas d'un conte (tärät). De ce point de vue, le passage incessant de T'äbəqäw d'un pronom à l'autre (du “il" au "tu”, puis au "nous", parfois au sein d'une même proposition) a pour effet d'accentuer cette universalité. Mais la vérité ainsi racontée s'arrêtait toujours au troisième temps, comme si l'histoire ne comportait pas de chute, et qu'on ne savait ce qu'il advenait des deux protagonistes. Ici, T'äbəqäw marque donc un temps d'arrêt ; et c'est Täsämma qui décide de le relancer sur les enjeux et la gravité de la rencontre:

Täsämma - D’accord. Ensuite, est-ce qu'on vous dit de ne pas jouer quand vous allez loin?

T’äbəqäw - Dans le désert. C'est-à-dire quand les vaches paissent dans le désert. Quand les vaches paissent dans le désert, ce qui les entoure c'est de la forêt, n'est-ce pas? Quand c'est de la forêt, on lui dit " ne joue pas" au moment où il sort.

Täsämma - C'est-à-dire qu'il [le léopard] pourrait peut-être blesser les vaches.

T’äbəqäw - Oui, oui. Et nous aussi, il nous blesserait.

Täsämma - Tout comme les vaches.

T’äbəqäw - Maintenant, alors que quelqu'un jouait comme ça aux vaches, il est venu. Il s'est propulsé en rampant et n’a pas réveillé...

Täsämma - Les vaches.

T’äbəqäw - L'homme.

Täsämma - Oh !

Täsämma a donc son idée sur la nature de cet enjeu : il le fait porter sur la sécurité des vaches et insiste à trois reprises. Mais T’äbəqäw le contredit : c'est bien de celle du joueur qu'il s'agit. Et pour l'illustrer, il s'engage dans le récit d'une anecdote :

T'äbəqäw - Oui. C'est-à-dire, on dit que le père et le fils étaient couchés. Mais ceci, c'est la vérité. 
Täsämma - On dit que c'est de l'Histoire dans notre pays.

T'äbəqäw - Voici ce que j'ai entendu dire : à ce moment-là, le père dort, le fils est assis et là ce fils n'a pas le choix et doit jouer, jouer. Quand il se réveille...

Täsämma - Il est en train de gratter.

T’äbəqäw - Celui-là, assis, a sa patte posée. Ce léopard, je veux dire. Après, comment peut-il l'atteindre, il va lui frapper et lui tuer son fils. Alors, il prend son fusil et tout doucement il se cache derrière un veau et avance, tu vois. Celui-là, il se contente de jouer du roseau.

Täsämma - Et aussi, le léopard est en train d'écouter calmement.

T'äbəqäw - Oui, le léopard est en train d'écouter. Et l'homme, lentement, lentement, le vise de sa balle. Et celui-là qui joue du roseau, n'est-ce pas?

Täsämma - Oui.

T'äbəqäw - Celui-là qui joue du roseau a dit que l'homme ne l'avait pas touché. Mais celui-ci [le léopard] quand il a voulu partir, il l'a abattu, il l'a tué.

Täsämma - Oui il l'a tué. Mais le fils, lui, va bien.

T’äbəqäw - Oui le fils va bien. Ensuite, le léopard est mort, à ce qu'on dit.

T’äbəqäw insiste sur la véracité de cette histoire, encouragé en cela par Täsämma qui souligne son implication en anticipant ou en répétant des éléments du récit. Tout comme celle d'Ashenafi Kebede, l'histoire comporte une chute, quoiqu'inversée. Mais elle n'est pas très claire sur le sort qui aurait été réservé au fils si le père n'avait pas été là, où s'il avait eu moins de présence d'esprit. Personne ne m'a jamais mentionné le risque d'essoufflement ; l'un des mes interlocuteurs insista un jour sur le risque d'être tué et mangé ; mais son frère fut catégorique : "non, si on s'arrête, le léopard ne mange pas". 8 Bref, au delà de l'anecdote dont on a entendu parler - une anecdote qui comporte un dénouement possible mais en rien canonique - personne ne semble certain de ce qui pourrait arriver lors de la rencontre, comme si seule cette image, celle du léopard appuyant sa patte sur une épaule, était véritablement significative.

Ce qui importe en effet n'est pas la fin de l'histoire, mais le risque très particulier qu'il y a à jouer dans la nature. En effet, nous sommes ici très loin du mythe d'Orphée, qui par sa flûte charmait et contrôlait les animaux sauvages ; il s'agit plutôt d'une emprise paradoxale du léopard sur l'homme, paradoxale car hors de tout contrôle aussi bien de l'homme que de l'animal. En ce sens, ni l'un ni l'autre n'ont le pouvoir de modifier la situation une fois enclenchée, et le musicien n'a d'autre choix que d'accepter le pouvoir de son instrument 
ou, si la peur le saisit, d'éviter d'en jouer et d'engager cette communication dangereuse.

La relation entre la flûte et le léopard n'est pas l'unique risque auquel doit faire face l'individu seul en forêt. La voix - le chant - est tout autant source de danger. Aux ennemis, voleurs et bandits, elle signale sa position et peut être un prélude à la confrontation. Réveillés à temps, les gardiens les font plutôt fuir à coups de cris ou de fusils ; mais ils ne sont pas rares ceux qui ont tué un voleur qui s'était trop approché. Pourquoi donc prennent-ils ce risque ? Pour y répondre, il nous faut s'attarder d'abord quelque temps sur cette figure du bandit.

\section{UNE VIE "SAUVAGE" : DE LA CONFRONTATION A LA TRANSFORMATION}

Cette partie de la vallée de l'Abbay est probablement la dernière héritière de la notoriété qui était celle de la région du XVIII ${ }^{\text {ème }}$ jusqu'au début du XX ${ }^{\text {mèe }}$ siècle. Durant ces deux siècles, et notamment dans les périodes de troubles politiques, elle fut particulièrement dangereuse : en tant que zone frontière entre les provinces du Goğğam et du Bägemdər, elle abritait alors un "banditisme endémique”, selon l'expression de Donald Crummey (1986: 136137). Selon les sources, telles que les chroniques royales, analysées par les historiens, des brigands y agissaient en bandes, s'attaquant aux caravanes au passage du gué tout proche. Le franchissement de la frontière, dans un sens ou dans l'autre, leur garantissait l'impunité, de même que le refuge de la forêt.

Ces "bad men of the borders" (Caulk 1984) présents sur l'ensemble des marges du royaume, sont considérés comme des acteurs à part entière de l'histoire éthiopienne, bien que les historiens aient débattu, dans les années 1980 et 1990, de la nature exacte de ce banditisme. Alors que le phénomène a d'abord été analysé comme une voie privilégiée d'accès au pouvoir politique pour les membres de la noblesse - comme le montre la carrière de Kassa, devenu l'empereur Tewodros (Crummey 1986: 139) - la perspective s'est élargie pour prendre en compte l'implication des paysans. Comme l'explique Fernyhough (1986), des individus pouvaient décider d'échapper à la pauvreté, et surtout à l'autorité de leurs seigneurs en rejoignant des bandes de hors-la-loi. Ces paysans étaient mus par une méfiance à l'égard du pouvoir dont ils partageaient cependant les valeurs. Ils se plaçaient ainsi à l'écart, mais non hors, du système ; et s'il n'existe plus aujourd'hui - du moins dans cette région - de bandes de brigands, la forêt continue à servir de refuge temporaire aux voleurs occasionnels et aux meurtriers.

L'attitude des paysans envers les hors-la-loi est assez ambiguë : elle oscille entre la réprobation et une idéalisation admirative dont Donald Levine écrit avec justesse qu'elle trouve son origine dans un modèle de virilité, d'autonomie et 
de courage très présent chez les Amhara. ${ }^{9}$ Alors même qu'on se désole de leur "méchanceté" (kofat), on les dépeint souvent comme des hommes braves et généreux : tel ancien paysan devenu fugitif était un "héros" (ğägna), qui chantait "tout le temps" seul la nuit, perché dans un arbre pour se protéger des hyènes. Ce sont des hommes "lésés" (yäkkäfaw), admirés pour leur opposition à l'autorité. Cette vision positive est particulièrement développée dans certains poèmes chantés :

\section{Dsat bällaw alu Gugube tärarawan muna dängälun}

Yäkäfaw wänd lăg yämiyast’ägawən
On dit que le feu a ravagé la montagne Gugube, les roseaux nains

Qui abritaient l'homme brave et lésé ${ }^{10}$

Nous ne sommes pas loin de ce qu'écrivait Hobsbawm (1972: 36-37) à propos de l'image du "bandit au grand cœur" :

- Ce sont ainsi des hommes vivant seuls, dans la "nature sauvage" qui leur sert de refuge. Ils mènent une existence désocialisée pour échapper aux recherches des autorités.

- Ils ne sont en aucun cas comparables aux voleurs ordinaires attirés par l'unique appât du gain, ces hommes ne sont pas crapuleux ; mus par des circonstances qui les dépassent (comme la pauvreté), ils sont tombés dans le malheur (mäkära) et n'ont d'autre choix que de voler pour survivre.

- Enfin le fusil - celui que, comme dans de nombreuses autres sociétés éthiopiennes, chaque homme possède - devient leur seul compagnon, leur seule famille. Leur vie est donc faite de violence, mais d'une violence parée de noblesse, car défensive, elle n'est destinée qu'à réparer le tort personnel qui leur a été causé.

Ces "héros" sont donc les meilleurs représentants d'une certaine vie "sauvage " - extérieure au village, à sa sociabilité, et aux obligations issues des règles de la parenté. ${ }^{11}$ Or on note, lors des séjours "aux vaches", un changement très sensible de perspective : au lieu de la menace et de la confrontation potentielle (dont nous avons vu qu'elles sont sans cesse rappelées au village), nous

9 "The attitudes of civilian Amhara towards the shifta combine fear and dislike with a strong tendency toward idealization. [...] He is guabaz, the great Amhara virtue that embodies bravery, fierciness, hardihood and general male competence" (Levine 1965: 244).

10 Chanté par Gännätu Bilaw.

11 Pour les questions de parenté, on peut se référer à Hoben (1973) et Morand (201 1, 2012). 
trouvons une identification voire une transformation. Il se passe en effet, au moment du chant, quelque chose de l'ordre de l'ensauvagement.

La nature "sauvage" (duru, dänu) est très souvent personnifiée et prise à témoin - "Monsieur Nature", lui dit-on. ${ }^{12}$ Deux figures animales sont aussi sollicitées. La première est justement celle du léopard, qui représente le héros en raison du courage et de l'indépendance qui lui sont communément attribués : on se souvient en effet des propos d'Ashenafi Kebede, rapportés plus haut, et de cette relation paradoxale qui s'établit par le truchement de la flûte. Il n'en est d'ailleurs pas qu'une simple représentation : en effet l'homme qui dort en forêt semble devenir symboliquement léopard, comme le souligne ce poème qui compare la voix de l'homme solitaire avec le rugissement de l'animal :

Olm kalläw čakka ১lm kalläw dur

Siyagwärämärämäw yadära läsäw әndä näbər
Dans la forêt profonde, dans la nature profonde

Hommes comme léopards, ils rugissent ceux qui y passent la nuit ${ }^{13}$

La deuxième figure est celle du bœuf, l'animal domestique par excellence et dont le retour à un état sauvage, celui de taureau - retour symbolique puisqu'il n'existe aucune espèce de bovidé sauvage en Éthiopie - est une métaphore de celui que pourrait entreprendre l'individu :

\section{Antä Šašä bäre botəhedom yarsuhal botstänam yarduhal}

Manqiñawn səbər duru yəšälahal
Toi le bœuf Šašä, quand tu marches ils t'obligent à labourer, quand tu te couches ils t'égorgent

Casse ta corde, la nature te convient mieux ${ }^{14}$

Dans ce type de poèmes, où le jeu des pronoms sans cesse interchangés - du “il", au "tu" et au "moi" - accentue l'identification des différentes figures, le chanteur se met lui-même en scène dans un appel à l'ensauvagement qui le libérerait de la société, achevant par le chant la métamorphose en bandit-héros que sa simple présence en forêt avait déjà initiée. Or cette métamorphose ne

12 Voir le poème en exergue de cet article. "Nature” est un substantif masculin en amharique. Il faut aussi souligner que cette personnification, spécifique aux poèmes chantés, est pour ainsi dire inexistante dans le langage ordinaire.

13 Chanté par Getačaw Assäfa.

14 Chanté par Gätənnät Gäbru. 
semble pas ici évitée par une musique, qui préserverait l'individu - selon l'hypothèse formulée par Seydou (1991) ou Spittler (2001) -, mais bien accentuée et menée par celle-ci à son terme.

Pourquoi cette transformation? Peut-on dire qu'il s'agit d'un désir de briser toute forme d'attachement social ? En réalité, le problème est un peu plus complexe que cela et il nous faut, pour y répondre, nous pencher plus en détail sur le statut de ces séjours "aux vaches".

\section{A DISTANCE : UNE FAUSSE SÉPARATION}

Tout d'abord, il est important de souligner que cette figure du "héros", bien qu'elle soit idéalisée, n'est pas une figure uniquement légendaire ou poétique. Sa réalité, contemporaine et toujours douloureuse, est bien connue. La vie d'un hors-la-loi en forêt s'avère en effet plus misérable que glorieuse : des discours de leurs proches - épouses, frères ou enfants - transparaissent surtout la faim, la maladie, et une forte dépendance envers la solidarité familiale ; les recherches qu'il faut affronter et les efforts de réintégration; et les conséquences considérables sur la vie sociale de leur cercle familial. Ainsi, le "héros" est à distance de la société sans en être en dehors pour autant. Il vit toujours son exclusion comme un fait temporaire, sans perdre l'espoir de revenir et de reprendre sa place. La forêt, inscrite dans le territoire tout en en formant la marge incontrôlée, constitue de ce point de vue l'espace idéal pour leur servir de refuge. La question est donc bien celle de l'espace et de la distance physique qu'il induit ; elle est aussi celle du temps. Et la place de l'élevage et de la garde du bétail soulève exactement les mêmes problèmes.

En effet, nous avons affaire à une société de cultivateurs, et qui se revendique comme telle. Comme tous les observateurs ont pu le souligner, les habitants des haut-plateaux Amhara se définissent eux-mêmes comme "paysans", gäbäre (littéralement "ceux qui paient l'impôt") ; et le terme de "labour" (ərša) désigne à lui seul un ensemble d'activités - le travail agricole - qui sont autrement plus valorisées que le commerce ou l'artisanat, par exemple.

La place de l'élevage est pourtant un peu plus ambiguë. On ne peut pas dire que pour ces "laboureurs" l'activité de garde à proprement parler soit valorisée. Mais pour un paysan cultivant ses propres terres, passer du temps avec ses "vaches" (lam) est une activité qui est plus respectable: il est même très mal vu de s'y dérober.

Dans cette société où les biens matériels sont peu nombreux et rarement distinctifs, un nombre élevé de vaches et de bœufs est le principal signe extérieur de richesse. Leur accumulation est donc un objectif primordial, qu'elle s'effectue par la voie de l'héritage, de l'achat, et du vol - un acte prestigieux. Richesse, statut social, et pouvoir : s'il n'est assurément pas la source principale de revenu, l'élevage de bétail est un aspect important de la vie locale. 
Simon Messing avait bien remarqué l'ambiguïté de ces sentiments : il voit dans les rapports des hommes à leurs animaux, et notamment dans l'existence de cette transhumance saisonnière, une "réminiscence d'un passé [...] semi-nomade" (Messing 1957: 119). Il est certain que certains traits, comme l'importance économique du bétail, ou le prestige du vol qui lui est associée, peuvent évoquer le fonctionnement des sociétés pastorales. Mais plutôt que d'en chercher les raisons dans un hypothétique passé (vieux de tant de siècles !), il me semble plus intéressant d'analyser cette ambivalence au prisme des difficultés et des contradictions de ce que James McCann (1995) a nommé, dans son ouvrage sur l'histoire agricole de l'Éthiopie, le "système de l'araire" (ou "ox-plow complex").

Dans ce système, le maintien de l'élevage est vital pour l'utilisation de cette technique agricole : en effet, l'araire nécessite au minimum deux bœufs, pour être manié ; et compte tenu du travail nécessaire au labour - l'araire ne permettant pas un retournement profond du sol, et nécessitant plusieurs passages pour être effectif -, une deuxième paire de bœufs est souvent nécessaire. Les paysans ont donc besoin d'entretenir des troupeaux suffisamment conséquents pour s'assurer d'avoir en permanence des animaux de trait disponibles. Le "système de l'araire" a donc toujours dépendu, conclut l'auteur, de l'habileté des paysans à maintenir leur cheptel malgré l'accroissement constant des surfaces cultivées.

Ce système est donc fondé d'un équilibre délicat entre le rythme annuel et donc irrégulier - imposé par la culture des céréales, et le temps et l'énergie à consacrer à un troupeau qu'il faut nourrir et soigner quotidiennement. À la fois complémentaires et en concurrence, les exigences contradictoires de ces deux activités peuvent expliquer les ambivalences envers le bétail. En périodes de labour ou de moissons les hommes doivent encore parvenir, alors qu'ils sont épuisés, à dormir tout en montant la garde de l'enclos en forêt ou en plein champ, contre les incursions potentielles de hyènes ou de voleurs.

Le calendrier agricole, succession de périodes de labour, de semis, de désherbage et de récolte des céréales et des légumineuses, est particulièrement contraignant. Le calendrier religieux ne l'est pas moins. Longtemps puissante aussi bien au niveau national que régional, l'Eglise orthodoxe a certes perdu une grande partie de son influence politique et de son pouvoir économique. Elle n'en garde pas moins une influence considérable sur les individus, et notamment sur leur cycle de vie (le baptême, éventuellement le mariage religieux, l'enterrement, et les commémorations funéraires, täzkar). Les obligations prennent la forme :

- d'un cycle hebdomadaire, où deux jours doivent être jeûnés (mercredi et vendredi), et deux jours chômés (samedi et dimanche), pendant lesquels 
il est interdit de "toucher", c'est-à-dire d'effectuer la plupart des travaux manuels, à l'exception de la garde des troupeaux;

- d'un cycle mensuel, où des fêtes de saints, à date fixe, doivent être chômées ;

- et d'un cycle annuel marqué par de longues périodes de jeûnes (dont le carême), et par la célébration des fêtes majeures, comme la fête de la Croix (Mäsqül), Noël (Gänna), l'Epiphanie (t’’mqät), Pâques (Fasika), et la célébration annuelle du saint patron de la paroisse (Amätzbal).

Aux calendriers agricole et religieux s'ajoute enfin le calendrier social, en grande partie déterminé par ces derniers. Les célébrations et les périodes de jeûnes marquent ainsi le début et la fin des deux saisons des mariages ; les samedis sont consacrés au marché et les jours chômés aux visites familiales. Le calendrier de la garde du bétail est donc un calendrier par défaut. A certaines périodes de l'année, les obligations agricoles, religieuses, et sociales laissent très peu de marge pour se rendre en forêt, par exemple, d'où le recours à la délibération collective ainsi qu'à des décisions personnelles - qui ne sont pas sans conséquences sur la perception et le vécu de cette activité.

\section{LE LIEU DE L'INTIME}

Ainsi, tout comme le bandit qui n'est à l'écart de la société que le temps qu'il lui faudra pour s'y réintégrer, les espaces et les temps “aux vaches" n'existent pas de manière autonome mais par défaut, autrement dit en référence à quelque chose d'autre qu'eux-mêmes : les lieux et moments de la sociabilité villageoise, auxquels les ramènent en permanence leurs pensées soucieuses. Des travaux des champs aux relations personnelles, elles se suivent et s'enchaînent: où en sont les labours? Quelle fête faut-il préparer et quels invités s'y présenteront? Comment s'imposer dans ce conflit de terre avec le voisin? Et si seulement cet oncle n'était pas décédé, les événements n'auraient-ils pas pris une tournure différente ? $^{15}$

Or c'est précisément la musique qui se fait le support de ces pensées. Ces dernières prennent le nom de tazata, un terme qui signifie littéralement "remémoration", "réminiscence", et désigne, dans cette région, une certaine disposition d'esprit qui pousse à chanter. Pour reprendre les mots de mon hôtesse Abäba, "la tazata vient dans la nature. Quand [les gens] sont seuls, qu'il n'y a autour d'eux que les arbres, l'eau, les animaux sauvages et les vaches qui broutent, et qu'il n'y a pas de maisons, la tazzta leur vient. Alors ils chantent". Mais elle désigne aussi le fil de souvenirs et d'images issues du passé qui accompagnent la pratique solitaire de la musique en forêt. 
Pourquoi des souvenirs? Parce que, comme je l'ai souligné plus haut, toutes les musiques "aux vaches" ou "en forêt" sont issues de la sociabilité villageoise - y compris la flûte, puisqu'une grande partie de son répertoire consiste en chants transposés et adaptés à l'instrument. Les Goğğamés, dans leur solitude en forêt, ne chantent rien de nouveau: ils réitèrent des chants qu'ils ont précédemment entendus (ou eux-mêmes chantés) lors de fêtes, de funérailles, ou encore de travaux collectifs. Lors de ce passage du village à la forêt, ces chants restent fortement associés à leur contexte initial (événements et personnes précis) et, un peu à la manière d'une madeleine de Proust, engendrent des souvenirs précis et extrêmement vivaces. ${ }^{16}$ Tous mes interlocuteurs affirment revivre l'instant où ils l'ont chanté ou entendu pour la première fois dans toute son intensité initiale: cette femme, par exemple, qui tout juste revenue du fleuve, se souvient de son explosion de colère lors de l'assassinat d'un de ses frères en rechantant son appel à la vengeance lancé devant le cercueil ; son cousin convoquant un soir l'image et la voix puissante de son père dans un souvenir d'enfance; ou encore son fils adolescent racontant un soir comment, plus tôt dans la forêt, au milieu des phacochères et des hyènes, la tazzta de son père absent lui était "venue", sous la forme d'une série d'instants différents, de jour comme de nuit, que tous deux avaient partagé. Ils lui étaient tous venus "d'un seul coup, avec leurs poèmes". Et il les aurait chantés, si cela n'avait été trop dangereux, en raison des voleurs - une crainte que son père, qui chantait tout le temps, n'aurait certainement jamais ressentie. ${ }^{17}$

De cette pratique de la musique qu'ils disent "privée" (yägal) ou "pour eux-mêmes" (läbəčča), les Goğğamés tirent un plaisir intime : assis les yeux baissés ou fermés, comme plongés dans un état de profonde concentration, ils s'enveloppent dans la grande pièce de coton $(k u t a)$, dont un pan vient parfois leur recouvrir la bouche - dans une posture qui rappelle curieusement celle que l'on observe chez les personnes fatiguées ou malades lorsqu'elles souhaitent s'isoler, à la maison, de la conversation environnante. Échos d'un autre temps (de quelques semaines à plusieurs décennies) ces chants de solitude sont l'occasion d'un retour sur soi-même, sur ses émotions et sur sa propre histoire qui ne se présente que rarement dans d'autres contextes. C'est dans l'isolement de la forêt, là où les dangers (prédateurs, voleurs, et ennemis potentiels) exigent paradoxalement une attention permanente, qu'éclosent les pensées vagabondes, entre pleurs et mouvements de colère.

16 Je me suis étendue longuement sur le concept de təzəta et sur les mécanismes de cette remémoration par la musique dans d'autres publications (Morand 2008, 2010, 2011).

17 Conversation avec Sisay Fasigaw. Il faut préciser que le père en question était alors en prison pour un double meurtre, après avoir vécu deux ans dans la forêt en tant que bandit. L'histoire de cette famille, telle qu'elle transparaît à travers la pratique du chant, est racontée en détail dans Morand (2012). 
Le "son de la forêt", tant apprécié des Goğğamés, est donc la matérialisation de leur rapport ambigu aux espaces qui entourent village et champs. Dans ces voix solitaires, souvent étouffées par une main ou un pan de vêtement et qui s'élèvent au-dessus des bruits de la forêt, ils reconnaissent un défi lancé aux dangers de la nature "sauvage", à ses prédateurs et à ses bandits auxquels ils sont souvent assimilés. Ce défi est celui de la maîtrise : par sa présence mobile et continue, la musique affirme une volonté de contrôle, voire de territorialisme. C'est le cas tout particulièrement des sifflets qui sont destinés au troupeau et signalent la position de leur gardien dans l'espace de la forêt. Or cette maîtrise n'est jamais acquise. Comme la rencontre potentielle de la flûte et du léopard le montre, la musique est une prise de risque aussi bien pour son intégrité physique que pour son identité en tant qu'être social. De là surgissent le trouble et l'inquiétude - premier pan, souligné avec insistance et emphase, de ce rapport aux espaces de la forêt.

Le second pan est beaucoup moins susceptible d'être évoqué en dehors d'un cercle de proche, et pour cause: il s'agit de ce sentiment d'intimité, de retrait sur "soi seul", sur ses souvenirs et sur ses émotions qui naît de la distance avec la sociabilité du village. Car celle-ci ne signifie pas que les affaires y sont oubliées, ou mises de côté; elles y prennent au contraire une importance considérable, quoique plus réflexive. Pas plus que les bandits, ces hommes n'expérimentent en effet de séparation totale. Vivant avant tout de la culture de leurs terres, ils ne sont que des bergers temporaires et les marges que sont ces espaces non cultivés et non contrôlés sont intrinsèquement liées au centre (champs et habitations), ce dernier dépendant de leur présence pour sa perpétuation. Le chant, en tant qu'écho de leur vie sociale, les relie en permanence à l'univers du village tandis qu'ils parcourent la forêt dans un double mouvement de tension vers l'extérieur et de repli sur soi, toujours aux aguets malgré le fil de leurs souvenirs. Né de l'inquiétude et du sentiment d'intimité, le “son de la forêt" devient, pour ceux qui l'entendent, l'indice de pensées les plus privées. 


\section{BIBLIOGRAPHIE ${ }^{18}$}

ASHENAFI KEBEDE, 197 1, The Music of Ethiopia: Its Development and Cultural Setting. Middletown, CT, Wesleyan University, thèse de doctorat.

BRUCE, James 2004 [1790-92], Voyage aux sources du Nil, 1769-1770. Paris, Karthala.

CAULK, Richard, 1984, "Bad men of the Borders: shum and shefta in Northern Ethiopia in the 19th century", The International Journal of African Historical Studies, 17 (2): 201-227.

CRUMMEY, Donald, 1986, "Banditry and resistance: noble and peasant in nineteenth-century Ethiopia”, in Donald Crummey (org.), Banditry, Rebellion, and Social Protest in Africa. Londres, James Currey, 133-149.

DE LANNOY, Michel, 1994, "La voix dans son espace", in Jacques Coget (org.), L'homme, l'animal et la musique. St.-Jouin-de-Milly, FAMDT Editions.

FERNYHOUGH, Timothy, 1986, "Social mobility and dissident elites in Northern Ethiopia: the role of Bandits, 1900-69", in Donald Crummey (org.), Banditry, Rebellion, and Social Protest in Africa. Londres, James Currey, 151-172.

HOBEN, Allan, 1973, Land Tenure among the Amhara of Ethiopia: The Dynamics of Cognatic Descent. Chicago, The University of Chicago Press.

HOBSBAWM, Eric, 1972, Les bandits. Paris, La Découverte.

LEVINE, Donald, 1965, Wax and Gold: Tradition and Innovation in Ethiopian Culture. Chicago, The University of Chicago Press.

McCANn, James, 1995, People of the Plow: an Agricultural History of Ethiopia. Madison, The University of Wisconsin Press.

MESSING, Simon, 1957, The Highland-Plateau Amhara of Ethiopia. Philadelphia, University of Pennsylvania, thèse de doctorat.

MORAND, Katell, 2008, "Chanter pour soi: La tïzïta du berger”, Annales d'Ethiopie, XXIII: 45-59.

MORAND, Katell, 2010, "Fragments de vies: quand les bergers se racontent par la musique", in G. D’Agostino, M. Kilani et S. Montes (orgs.), Histoires de vies, témoignages, autobiographies de terrain: Formes d'énonciation et de textualisation. Munich, Lit Verlag, 257-272.

MORAND, Katell, 2011 , “Mélodies pleurées, paroles à attraper: les chants d'angurguro dans des funérailles éthiopiennes", Cahiers de Littérature Orale, 69: 57-78.

MORAND, Katell, 2012, Solitudes habitées: Le chant, le souvenir et le conflit chez les Amhara du Goğğam (Éthiopie). Nanterre, Université Paris Ouest Nanterre La Défense, thèse de doctorat.

SEYDOU, Christiane, 1991, Bergers des mots : poésie peule du Mâssina. Issy-les-Moulineaux, Les Belles Lettres.

SPITTLER, Gerd, 2001, "Le berger et la solitude", in Papa Samba Diop et Hans-Jürgen Lusenbrick (orgs.), Littératures et sociétés africaines: Regards comparatistes et perspectives interculturelles. Tübingen, G. Narr Verlag, 219-228.

18 Selon l'usage, les auteurs éthiopiens sont indexés par leur prénom suivi du prénom de leur père. 